\title{
Bipolar and Related Disorders Induced by Sodium 4-Phenylbutyrate in a Male Adolescent with Bile Salt Export Pump Deficiency Disease
}

\author{
Giovanni Vitale ${ }^{1}$, Giulia Simonetti ${ }^{1}$, Martina Pirillo ${ }^{1}$, Gianfranco Taruschio ${ }^{2}$, and Pietro Andreone ${ }^{1}$ \\ ${ }^{1}$ Department of Medical and Surgical Sciences, University of Bologna, Bologna, Italy \\ ${ }^{2}$ Department of Mental Health, SPDC Unit, USL Local Health Bologna, Bologna, Italy
}

Bile Salt Export Pump (BSEP) Deficiency disease, including Progressive Familial Intrahepatic Cholestasis type 2 (PFIC2), is a rare disease, usually leading within the first ten years to portal hypertension, liver failure, hepatocellular carcinoma. Often liver transplantation is needed. Sodium 4-phenylbutyrate (4-PB) seems to be a potential therapeutic compound for PFIC2. Psychiatric side effects in the adolescent population are little known and little studied since the drug used to treat children and infants. So we described a case of Caucasian boy, suffering from a late onset PFIC2, listed for a liver transplant when he was sixteen and treated with 4-FB (200 mg per kilogram of body weight per day). The drug was discontinued for the onset of bipolar and related disorders. This case illustrates possible psychiatric side effects of the drug.

Psychiatry Investig 2016;13(5):580-582

Key Words Bipolar and related disorders, Sodium 4-phenylbutyrate, Progressive Familial Intrahepatic Cholestasis type 2.

\section{INTRODUCTION}

PFIC are an heterogeneous group of autosomal recessive disorders of childhood that present with intrahepatic cholestasis, characterized by defects in biliary proteins, involved in the synthesis and transport of bile1, leading to portal hypertension, liver failure and transplantation within first ten years of life. Up to now three different type of PFIC have been identified: type 1, 2 and 3. PFIC2, also known as BSEP deficiency disease, involves $\mathrm{ABCB} 11$ gene, encoding the bile salt export pump (BSEP), a liver-specific adenosine triphosphate binding cassette transporter that mediates the excretion of bile monovalent salts. ${ }^{2}$ Current treatments available are high-dose of ursodeoxycholic acid (25-30 mg/kg/die) in initial management, rifampicin for itching and fat-soluble vitamins (A, K, D, E) supplementation. ${ }^{2}$ Some patients with PFIC2 may also ben-

Received: July 1, 2015 Revised: December 18, 2015

Accepted: December 28, 2015 Available online: April 6, 2016

$\triangle$ Correspondence: Giulia Simonetti, $\mathrm{PhD}$

Dipartimento di Science Mediche e Chirurgiche, Università di Bologna, Via Massarenti 9, 40138 Bologna, Italy

Tel: +39 0512144873, +39 0512143152 Fax: +39051345806

E-mail: simgiu@hotmail.it

(c) This is an Open Access article distributed under the terms of the Creative Commons Attribution Non-Commercial License (http://creativecommons.org/licenses/bync/3.0) which permits unrestricted non-commercial use, distribution, and reproduction in any medium, provided the original work is properly cited. efit from biliary diversion, and endoscopic nasobiliary drainage, maintained for a few weeks, may help to select potential responders to biliary surgery. ${ }^{2}$ If these medical and surgical therapies fail, liver transplantation remains the only alternative treatment. ${ }^{3}$ 4-PB is an orphan drug (AMMONAPS; Swedish Orphan $A B$ Inter, Stockolm, Sweden), routinely used to treat ornithine trans-carbamylase deficiency (OTC). In last years, reports in vitro and in vivo, emerged about the effective use of the 4-PB in PFIC, able to improve liver tests and itching by increasing the hepatocanalicolar expression of BSEP and the biliary excretion capacity of bile salts. ${ }^{4,5}$ Dosage was $500 \mathrm{mg}$ per kilogram of body weight per day or total dose of 10 grams per day. ${ }^{6,7}$ Results of clinical trials have shown that 4-PB has few side effects and it is safe for patients: grade 3 neuro-cortical toxicity, consisting of excessive somnolence and confusion, was sudden, dose-dependent, accompanied by metabolic changes (hyponatremia and hypocalcemia) and reversible after discontinuation of the drug. ${ }^{8}$ Even when pilot studies were conducted in patients with cystic fibrosis or thalassemia, severe adverse events were not reported., ${ }^{9,10}$ There are limited information on side effects of long-term administration of drug to patients with OTC deficiency. In this case, the most common adverse event was amenorrhea in $23 \%$ of post-pubertal females treated; other problems included anorexia and vomiting. ${ }^{11}$ When $4-\mathrm{FB}$ was used in patients with PFIC2, no severe 
side effects were observed during or after these studies. ${ }^{4,6,12} \mathrm{Fi}$ nally, the leaflet of the drug reported only two common psychiatric adverse effects, depression and irritability. Considering OTC deficiency provides per se to the development of neurological symptoms such as lethargy and irritability, related to hyperammonemia, the adverse events are to be analysed in this clinical setting. ${ }^{13}$

\section{CASE}

Mr. N., a Caucasian boy of 18 years, suffering from a genetically diagnosed late onset PFIC2, dyslexia and dyschromia, was listed for a liver transplant in January 2013 without evidence of psychiatric contraindications or mental disorders, detected during a specialist evaluation, performed as part of the examinations for inclusion in the waiting list.

From August 2014, he presented a recurrent jaundice with progressive increase in total bilirubin (until to $14.5 \mathrm{mg} / \mathrm{dL}$ ) without liver failure. Ultrasounds and endoscopy excluded complications such as ascites, esophageal varices and portal vein thrombosis. An off-label treatment with 4-PB was started at the initial dose of $200 \mathrm{mg}$ per kilogram of body weight per day, refracted in four times. Concomitant therapies were ursodeoxycholic acid (25 mg per kilogram of body weight per day), rifampin, taken for the itching (300 $\mathrm{mg}$ per day) and regular supplementation of fat-soluble vitamins (A, D, E, K). Systematically, after taking the drug, the patient complained dysphoria with uncontrolled irritability.

Laboratory tests didn't show electrolyte or metabolic abnormalities and infections were excluded. These manifestations caused a significant negative impact on his quality of life and on interpersonal relationship. Furthermore the family referred that $\mathrm{Mr} \mathrm{N}$. appears very different and showed behaviors related to other-directed verbal aggressiveness, never occurred before taking the drug, repeated consistently after each 4-PB intake. In particular, the patient reported that after 30 minutes of the drug administration, he felt a sense of "nervous tension" that could not control nor to manage with coping activities. After these episodes, intense asthenia and fatigue were reported. Then, recurrent headache and nausea and, occasionally, feeling of retrosternal weight with features not suggestive of angina were also complained. After two weeks, the treatment was discontinued and a psychiatric consultation was requested. A comprehensive psychiatric evaluation was performed by administering the Toronto Alexitymia Scale (TAS-20) ${ }^{14}$ (cut off scores for psychopatology >50), the State Trait Anger Expression Inventory (STAXI-II) ${ }^{15}$ (range of normality between $25^{\circ}$ and $75^{\circ}$ percentile), the Disability Scale and the Structured Clinical Interview for DSM-IV Axis II Disorders (SCID-II). ${ }^{16}$ For an initial analysis, the psychodiagnostic exam provided few relevant elements. In fact we excluded the alexitymic disease for TAS score $<50$ and $5 / 8$ subscale of the STAXI-II presented subthreshold scores $\left(<25^{\circ}\right.$ percentile). After careful analysis of the STAXI-II, we note very much subthreshold scores but out of the range for the subscale concerning the control of anger ( $\mathrm{Ax} / \mathrm{Con}>75^{\circ}$ percentile). These results reported a picture of a boy who may use excessively psychological defenses such as negation and repression that prevent uncomfortable or unacceptable feelings of anger. Mr. N. tended to invest a lot of energy controlling and preventing the experience and expression of the anger. He referred an ego-dystonic perception of the behaviors acted out. Even if not confirmed by SCID II scores, ${ }^{16}$ the hypothesis of a passive-aggressive personality disorder emerged clearly and the psychiatric symptoms completely regressed immediately after drug discontinuation. The interview revealed that the study activities was strongly influenced by the disease, however considering the diagnosis of dyslexia, instead the social and family life have not been affected by the health problem. Despite these psychological analyses, were not present evident symptoms of psychopathology, so the psychiatric evaluation excluded the presence of mental disorders in progress. The absence of disturbance in attention and awareness, an additional disturbance in cognition, finally, psychomotor behavioral disturbances ruled out alternative diagnosis of delirium. Instead, according with the DSM5 criteria, ${ }^{17}$ the psychiatric manifestations occurred in this boy were suggestive of bipolar and related disorders induced by $4-\mathrm{PB}$; the clinical features are: A) a significant and persistent altered mood that dominates the clinical situation, characterized by irritable mood; B) the symptoms developed immediately after drug exposure and this drug can produce psychiatric symptoms; C) the disturb is not explained by a bipolar disorder or related disorders not induced by drugs; $\mathrm{D}$ ) the disturbance does not occur during a delirium; E) alteration causes clinically significant distress.

\section{DISCUSSION}

4-PB is a chaperone orphan drug that has been recently indicated for the treatment of BSEP deficiency disease ${ }^{6,7}$ because is able to improve the quality of life through the reduction of itching, the episodes of jaundice and the need for invasive treatment, including liver transplantation. Psychiatric side effects as depression and irritability have been reported but no cases of bipolar and related disorders have been described until now in patient with PFIC2. We do not know the influence of personality traits and psychological factors on the development of symptoms, but can hypothesize in this case the presence of a psychological predisposition to a dysfunctional functioning concerning the expression of anger. So it is possible 
that this drug has also amplified a silent psychological tendency. Moreover, the drug obviously has effects other than just being a substrate for an alternate pathway of ammonia excretion and because it penetrate well into cerebrospinal fluid. ${ }^{18}$ Also, 4-FB was been studied in many diseases affecting the CNS such as spinal muscular atrophy, multiple sclerosis and cerebral ischemia where it seems to have helped, being able to slow cell apoptosis. ${ }^{19}$

Early researches showed that bipolar disorder is accompanied by the activation of immune-inflammatory pathways as indicated by the increased levels of pro-inflammatory cytokines, positive acute-phase proteins, such as multiple changes in oxidative and nitrosative stress, tryptophan and tryptophan catabolites, neurotrophins, circadian dysregulation, hypothalamic pituitary adrenal, axis dysregulation activation markers. ${ }^{20,21}$

The drug crosses the blood-brain barrier and influences the brain activity in different ways including tryptophan metabolism, ${ }^{22-24}$ but the exact mechanisms of the 4-PB related CNS adverse effects, have not yet been identified.

New studies should investigate aspects of neuropharmacology and possible relationships with the psychological aspects of subjects taking this drug. This is the first report documenting other psychiatric manifestations related to 4 - $\mathrm{PB}$ administration, recurring regularly after re-taking the drug, and reinforce the need of a careful psychiatric evaluation before starting the treatment in patients with PFIC.

\section{REFERENCES}

1. Morotti RA, Suchy FJ, Magid MS. Progressive familial intrahepatic cholestasis (PFIC) type 1, 2, and 3: a review of the liver pathology findings. Semin Liver Dis 2011;31:3-10.

2. Hori T, Nguyen JH, Uemoto S. Progressive familial intrahepatic cholestasis. Hepatobiliary Pancreat Dis Int 2010;6:570-578.

3. Soubrane O, Gauthier F, DeVictor D, Bernard O, Valayer J, Houssin D, et al. Orthotopic liver transplantation for Byler disease. Transplantation 1990;50:804-806.

4. Hayashi H, Sugiyama Y. 4-phenylbutyrate enhances the cell surface expression and the transport capacity of wild-type and mutated bile salt export pumps. Hepatology 2007;45:1506-1516.

5. Hasegawa Y, Hayashi H, Naoi S, Kondou H, Bessho K, Igarashi K, et al. Intractable itch relieved by 4-phenylbutyrate therapy in patients with progressive familial intrahepatic cholestasis type 1. Orphanet J Rare Dis 2014;9:89.

6. Naoi S, Hayashi H, Inoue T, Tanikawa K, Igarashi K, Nagasaka H, et al. Improved liver function and relieved pruritus after 4-phenylbutyrate therapy in a patient with progressive familial intrahepatic cholestasis type 2. J Pediatr 2014;5:1219-1227.

7. Gonzales E, Grosse B, Cassio D, Davit-Spraul A, Fabre M, Jacquemin E. Successful mutation specific chaperone therapy with 4-phenylbutyr- ate in a child with progressive familial intrahepatic cholestasis type $2 . J$ Hepatol 2012;57:695-698.

8. Carducci MA, Gilbert J, Bowling MK, Noe D, Eisenberger MA, Sinibaldi $\mathrm{V}$, et al. A phase I clinical and pharmacological evaluation of sodium phenylbutyrate on an 120-h infusion schedule. Clin Cancer Res 2001;7:3047-3055.

9. Rubenstein RC, Zeitlin PL. A pilot clinical trial of oral sodium 4-phenylbutyrate (Buphenyl) in Delta F508-homozygous cystic fibrosis patients: partial restoration of nasal epithelial CFTR function. Am J Respir Crit Care Med 1998;157:484-490.

10. Olivieri NF, Rees DC, Ginder GD, Thein SL, Brittenham GM, Way JS, et al. Treatment of thalassemia major with phenylbutyrate and hydroxyurea. Lancet 1997;350:491-492.

11. Burlina AB, Ogier H, Korall H, Trefz FK. Long-term treatment with sodium phenylbutyrate in ornithine transcarbamylase-deficient patients. Mol Genet Metab 2001;72:351-355.

12. Gonzales E, Grosse B, Schuller B, Davit-Spraul A, Conti F, Guettier C, et al. Targeted pharmacotherapy in progressive familial intrahepatic cholestasis type 2: Evidence for improvement of cholestasis with 4-phenylbutyrate. Hepatology 2015;62:558-566.

13. European Public Assessment Report of Ammonaps, European Medicine Agency, publishing on http://www.ema.europa.eu/docs/it_IT/ document_library/EPAR_Product_Information/human/000219/ WC500024753.pdf4. Accessed on Sep 11, 2009.

14. Bressi C, Taylor G, Parker J, Bressi S, Brambilla V, Aguglia E, et al. Cross validation of the -159-factor structure of the 20-item Toronto Alexithymia Scale: an Italian multicenter study. J Psychosom Res 1996;41:551-559.

15. Spielberger CD. STAXI-2: State-Trait Anger Expression Inventory-2: Manual / Adaptation Italian, at Anna Laura Comunian Care. Florence: Special Organizations; 2004.

16. Mazzi F, Morosini P, De Girolamo G, Guaraldi GP. SCID-II. O.S. The Italian Version. Florence: Special Organizations; 2003.

17. American Psychiatric Association. Diagnostic and Statistical Manual of Mental Disorders, Fifth Edition. Arlington, VA: American Psychiatric Publishing; 2013.

18. Berg S, Serabe B, Aleksic A, Bomgaars L, McGuffey L, Dauser R, et al. Pharmacokinetics and cerebrospinal fluid penetration of phenylacetate and phenylbutyrate in the nonhuman primate. Cancer Chemother Pharmacol 2001;47:385-390.

19. Qi X, Hosoi T, Okuma Y, Kaneko M, Nomura Y. Sodium 4-Phenylbutyrate protects against cerebral ischemic injury. Mol Pharmacol 2004;66: 899-908.

20. Anderson G, Maes M. Bipolar disorder: role of immune-inflammatory cytokines, oxidative and nitrosative stress and tryptophan catabolites. Curr Psychiatry Rep 2015;17:8.

21. Liu M, Brusilow WS, Needleman R. Activity of the yeast Tat2p tryptophan permease is sensitive to the anti-tumor agent 4-phenylbutyrate. Curr Genet 2004;46:256-268.

22. Grzanowski A, Needleman R, Brusilow WS. Immunosuppressant- like effects of phenylbutyrate on growth inhibition of Saccharomyces cerevisiae. Curr Genet 2002;41:142-149.

23. Cho JW, Choi SR, Hwang SG. Pharmaceutical composition for prevention and treatment of drug or alcohol addiction or bipolar disorder using sodium phenylbutyrate. U.S. Patent No 7,968,604, 2011.

24. Kulkarnia NN, Yia Z, Huehnkena C, Agerberthb B, Gudmundssona GH. Phenylbutyrate induces cathelicidin expression via the vitamin D receptor: Linkage to inflammatory and growth factor cytokines pathways. Mol Immunol 2015;63:530-539. 